\title{
MANGICULTURA E VALE DAS FRUTAS: QUAIS AS CONTRIBUIÇÕES CIENTÍFICAS DA ENGENHARIA DE PRODUÇÃO?
}

\author{
Bárbara Évelin Oliveira Sampaio* \\ Fernanda Roda de Souza Araújo Cassundé** \\ Nildo Ferreira Cassundé Junior ${ }^{* * *}$
}

RESUMO: Poucos setores possuem uma representatividade econômica com tamanha importância como o agronegócio. A fruticultura, por sua vez, tornou-se um interessante objeto de estudo tendo em vista crescentes demandas nacionais e internacionais, não apenas a partir da perspectiva de produção, como também do consumo. O Vale das Frutas e, especificamente, o cultivo da manga, exercem, nesse contexto, um papel importante em razão do desempenho de produção agrícola alcançado. Este estudo é, portanto, motivado pela seguinte questão norteadora: como os acadêmicos estão estudando a mangicultura, no contexto do Vale das Frutas, na área de Engenharia de Produção? Nessa perspectiva, este estudo concentrou esforços metodológicos capazes de revelar o estado do conhecimento da área. Como fontes de pesquisas para esta discussão optou-se em estudar periódicos classificados no Qualis/Capes 2014 genuínos da área de Engenharia de Produção no estrato B5 ou superior, assim como os anais do ENEGEP. A análise dos resultados, a partir dos critérios adotados, revela que a produção científica brasileira sobre mangicultura no Vale das Frutas, na área de Engenharia de Produção, é bastante pontual e, em alguns casos, como em periódicos, surpreendentemente inexistente.

PALAVRAS-CHAVE: Bibliometria; Estado do conhecimento; Fruticultura; Vale do São Francisco.

\section{MANGO-CULTURE AND THE FRUIT VALLEY: WHICH ARE THE SCIENTIFIC CONTRIBUTIONS OF PRODUCTION ENGINEERING?}

ABSTRACT: Agribusiness is perhaps one of the few segments with high relevant economic representativeness. Fruit culture has become an interesting study object

\footnotetext{
Graduanda em Engenharia de Produção pela Universidade Federal do Vale do São Francisco (UNIVASF).

** Doutora em Administração (UFPE); Docente Adjunta I do colegiado de Administração da Universidade Federal do Vale do São Francisco (UNIVASF); Docente permanente do Mestrado Profissional em Administração Pública (PROFIAP/UNIVASF); Coordenadora do Programa de Pós-Graduação em Dinâmicas de Desenvolvimento do Semiárido, Brasil; E-mail: fernanda.roda@univasf.edu.br

*** Doutor em Administração (PROPAD/UFPE 2015); Docente Adjunto I da Universidade Federal do Vale do São Francisco (UNIVASF).
} 
due to national and international demands. The Fruit Valley and especially mango cultivation have an important role due to their performance in agricultural products. The underlying question that motivated current analysis is: How do scientists study the culture of mangoes in Production Engineering, within the context of the Fruit Valley? Current analysis concentrated methodological efforts on the state-of-the-art. Research sources comprised journals in Qualis/Capes 2014 in the area of Production Engineering listed B5 or higher, and the ENEGEP annals. Results reveal that Brazilian scientific production on the culture of mangoes in the Fruit Valley, within the context of Production Engineering, is highly specific and in some case surprisingly non-existing.

KEY WORDS: Bibliometry; State of knowledge; Fruit culture; Vale do São Francisco.

\section{INTRODUÇÃO}

O agronegócio figura para o contexto mundial como um setor estratégico e merecedor de atenção. É tratado como uma política de Estado, já que barreiras protecionistas são impostas no sentido de proteger a economia local. Assim, é pouco provável imaginar um país se sustentar sem o auxílio da balança do agronegócio em sua economia. Aquelas nações que não são autossuficientes em seu abastecimento ou que não possuem terras agricultáveis suficientes para produzir determinados produtos da cadeia alimentar, inevitavelmente, precisam de nações parceiras para desempenhar tal função (OECD-FAO, 2015).

Nesse contexto, a fruticultura brasileira tem obtido relativo sucesso devido à disponibilidade de tecnologias, ao surgimento de novos mercados e à redução de barreiras comerciais (PIMENTEL; ALVES; FILGUEIRAS, 2000). Segundo o Ministério da Agricultura, Pecuária e Abastecimento (MAPA, 2012), em consonância com Siqueira (2003), a produção de frutas, especialmente nas áreas de semiárido, vem se apresentando como um dos setores que têm contribuído decisivamente para o crescimento econômico do Nordeste brasileiro enquanto região exportadora de frutas. Em razão das conquistas de mercado externo, tem-se um aumento de área plantada (IBGE, 2012), melhoria da qualidade da produção e da tecnologia usada pelo setor, além de maior profissionalização na etapa da comercialização. Destacam- 
se também o aumento da geração de renda, empregos e divisas, como ressaltam Vitti et al. (2004).

Assim como o Vale do Silício (Silicon Valley), situado no Estado da Califórnia/ EUA, é considerado o Polo Industrial que concentra diversas empresas de Tecnologia da Informação, o Vale das Frutas, situado no Submédio do Vale do São Francisco/BR (e que tem como cidades centrais Petrolina, no Estado de Pernambuco, e Juazeiro, no Estado da Bahia) é considerado o Polo Fruticultor do Brasil (LOPES; OLIVEIRA, 2012; SILVA-MATOS et al., 2014).

Responsável por quase a totalidade das mangas e uvas brasileiras de mesa exportadas, o Vale das Frutas, que já passou por diversos ciclos econômicos (GALVÃO, 2010), é reconhecido internacionalmente e faz do Brasil um importante player em termos de competitividade e eficiência na produção de frutas para exportação (JESUS JUNIOR; SIDONIO; MORAES, 2011; QUEIROZ et al., 2012).

As peculiaridades do Vale atraíram, portanto, empreendedores individuais e grandes grupos empresariais para investirem em uma produção fortemente marcada pelo capital intensivo, na medida em que a tecnologia de irrigação e o sistema de certificações aparecem ao lado das características climáticas enquanto vantagens competitivas valiosas em relação aos variados nichos de concorrência (CASSUNDÉ JR; LIMA; PIMENTEL, 2006).

Nesse sentido, diversas são as características das empresas atuantes no agronegócio especialmente sob a perspectiva da irrigação no Vale das Frutas (EMBRAPA, 2015). Sendo possível identificar, ao menos, três grupos distintos: (1) no que se refere ao volume produzido - dos pequenos aos grandes produtores, a exemplo do ex-Grupo Carrefour (EXSA, 2013); (2) no que concerne ao mercadoalvo: de fazendas que focam sua produção para abastecimento local (pequenos produtores dos Perímetros Irrigados) e/ou para o abastecimento internacional, a exemplo da Cooperativa COANA (COANA, 2013); e (3) os que foram criados exclusivamente para escoar a produção para o mercado internacional, vocacionadas para o mercado internacional, a exemplo da ARA Agrícola (ARA, 2015).

Dada a importância, portanto, do agronegócio e da mangicultura para o Vale das Frutas, este artigo é construído na tentativa de se resgatar o que tem sido pesquisado sobre o assunto. Nesse sentido, estudos que se propõem a delimitar 
o estado do conhecimento de determinada área são capazes de permitir mapear e identificar as ideias que estão sendo discutidas na academia. Assim, este estudo foi guiado pelo seguinte questionamento: Como os acadêmicos estão estudando a mangicultura, no contexto do Vale das Frutas, na área de Engenharia de Produção?

É importante destacar que uma decisão dos autores que limita o escopo do artigo reside no foco de análise, o contexto em Engenharia de Produção. Essa restrição não considerou os possíveis artigos de áreas afins, por exemplo, da Administração, da Engenharia Agrícola, da Economia.

No entanto, o foco dado na Engenharia de Produção justifica-se devido à estreita relação do tema, já que esta área trata do projeto, aperfeiçoamento e implantação de sistemas integrados de pessoas, materiais, informações, equipamentos e energia, para a produção de bens e serviços, de maneira econômica. Assim, considerando que as políticas de modernização da agricultura, no então polo irrigado Petrolina/Juazeiro, foram iniciadas na década de 1960 (OLIVEIRA; OLIVEIRA, 2012) para as culturas tradicionais de ciclo curto (SILVA, 2001), é possível dizer que o desenvolvimento do semiárido nordestino, de modo especial a Região do Vale do São Francisco, depende, em larga escala, da capacidade de seu parque industrial, de modo especial a fruticultura e vinicultura, de avançar em direção a maior qualidade e produtividade. Os meios e as ferramentas para atingir esses objetivos configuram a linha mestra de estudo da Engenharia de Produção.

\section{REFERENCIAL TEÓRICO}

\subsection{O VALE DAS FRUTAS}

Região banhada pelo Rio São Francisco, com $2.700 \mathrm{~km}$ de extensão, o Vale das Frutas abrange os Estados de Alagoas, Bahia, Minas Gerais, Pernambuco e Sergipe (ALVES, 2002). Atualmente, sua área, que é apropriada para irrigação, permite o beneficiamento de diversas culturas (CODEVASF, 2010). De características temporárias ou permanentes, destacam-se as de arroz, feijão, milho, melancia, cebola, abóbora e mandioca (temporárias); e manga, banana, cana-de-açúcar, coco, goiaba, uva, vinho e vinagre e acerola (permanentes). 
De todos os Estados que compõem o Vale das Frutas, os principais produtores estão localizados em Petrolina (Pernambuco) e Juazeiro (Bahia), como destaca Alves (2002). A atividade econômica está centrada na agricultura irrigada (CBHSF, 2012), constituindo-se, atualmente, a principal região exportadora de frutas frescas do país, especialmente uva e manga.

Possui uma boa infraestrutura de comercialização e serviços, já que, dentre as indústrias instaladas na região, destacam-se as processadoras de matéria-prima, de implementos e equipamentos de irrigação (BANCO DO NORDESTE, 1999). A expansão inicial do dipolo Petrolina/Juazeiro foi impulsionada, segundo Mariano (1999, p. 2), em função "dos pesados investimentos feitos pelo Governo Federal em infraestrutura física com implantação de gigantescas obras de irrigação".

A fruticultura no Polo Petrolina/Juazeiro se expressa em um conjunto de atividades inter-relacionadas, desde de muito tempo, constituindo uma cadeia produtiva com certo grau de complexidade, principalmente em função da diversidade: "a) de atores presentes no espaço produtivo; b) de culturas produzidas; c) dos mercados consumidores; e d) das formas de organização dos produtores" (BANCO DO NORDESTE, 1999, p. 33).

Considerando, portanto, as localizações de Petrolina (PE), de Juazeiro (BA) e da Bacia do Submédio do São Francisco, entende-se que a expressão Vale das Frutas torna-se mais apropriada à região que, atualmente, é comumente denominada, por muitos autores, como Polo Juazeiro/Petrolina (LANDIM; ALENCAR, 2013; MAT'TOSINHO et al., 2013; FREITAS; RUPOLO; OLIVEIRA, 2014) ou Submédio do Vale do São Francisco (GALVÃO, 2010; SILVA et al., 2012; BARBOSA; FERNANDES; LAGE, 2013; RIBEIRO et al., 2013).

A denominação Vale das Frutas, portanto, merece ser adotada em razão dos seguintes entendimentos:

a) a expressão "Polo Petrolina/Juazeiro" ou, simplesmente, "Juazeiro/ Petrolina" não mais efetivamente responde pela economia movimentada na região de seu entorno, pois muitas outras cidades circunscritas a Petrolina, no território pernambucano, e a Juazeiro, no território baiano, contribuem para que a região tenha sido evidenciada como um reconhecido caso de sucesso internacional (QUEIROZ et al., 2012);

b) a expressão "Submédio do Vale do São Francisco" é ampla demais para representar a realidade da produção do agronegócio da região. 


\subsection{A MANGICULTURA}

A expansão da Mangicultura ocorreu, inicialmente, no Estado de São Paulo, de onde foram difundidas as novas variedades para o restante do país, e nos polos de agricultura irrigada do Nordeste, onde houve a incorporação de plantios tecnificados, principalmente no Submédio do Vale do São Francisco e outras áreas irrigadas como as dos Vales do Jaguaribe, Açu-Mossoró, Parnaíba e Platô de Neópolis (EMBRAPA, 2010).

No ano de 2014, a manga tornou-se a fruta número um do ranking das exportações nacionais em termos de receita. Ela caiu nas graças dos consumidores brasileiros e internacionais especialmente depois que as ações de melhoramento genético resultaram em variedades não fibrosas, mais macias e saborosas, nos últimos 15 anos (SANTOS et al., 2014).

Em 2015, a maior receita também foi obtida com o embarque de 156,337 mil toneladas de manga. Tal resultado proporcionou alta de $17,52 \%$ em toneladas e de $12,59 \%$ em valor em relação ao verificado no ano anterior. O Vale das Frutas representou $84 \%$ das exportações brasileiras de manga em 2015 e seus maiores clientes são União Europeia e Estados Unidos, sendo o restante pulverizado entre Canadá e outros países (TREICHEL et al., 2016).

A manga, na região em análise, é explorada tanto pelo segmento da agricultura empresarial, representada pelas grandes, médias e pequenas empresas agrícolas localizadas nos diversos perímetros públicos de irrigação ou nas margens do Rio São Francisco, como também pelos produtores familiares, assentados nas áreas de colonização destes mesmos perímetros irrigados (EMBRAPA, 2009). Entre as variedades de manga, são produzidas a Tommy, Aktins, Keitt, Haden e Palmer (SNA, 2015).

Percebida a relevância do agronegócio, da fruticultura e da mangicultura para o Brasil e para região em destaque, estudos e pesquisas científicas que projetam e desenvolvem temáticas relacionadas são de grande influência para o crescimento e a inovação nesta área. Para o Ministério da Agricultura, Pecuária e Abastecimento (MAPA, 2007), é necessário construir uma sistemática e inovadora compreensão sobre o assunto, no intuito de abrir novas portas para todos os envolvidos nos setores institucional e acadêmico que procuram um conhecimento mais detalhado, 
objetivo e oportuno da agricultura e do mundo rural do país.

Assim, as produções científicas que abordam a temática em destaque contribuem para geração de base de dados e conhecimentos sobre as mudanças e evoluções no cenário do agronegócio e elementos correlacionados a ele. Conforme o MAPA (2007), conhecer os principais entraves e desafios do agronegócio de maneira séria, oportuna e sistêmica permitirá elevar a qualidade de insumos essenciais para a tomada de decisões e a formulação de políticas públicas mais eficientes.

\subsection{POR QUE É IMPORTANTE UM ESTUDO SOBRE O ESTADO DO CONHECIMEN- TO?}

Considerando os avanços e expansão da ciência e tecnologia nas últimas décadas, diversos são os autores que têm debatido a importância do mapeamento e discussão da produção acadêmica nas mais distintas áreas do conhecimento (FERREIRA, 2002; VANTI, 2002; CARDOSO et al., 2005; ARAÚJO, 2006; ARAÚJO; ALVARENGA, 2011; CASSUNDÉ; CASSUNDÉ JUNIOR, 2012). Assim, na medida em que a ciência vai se estabelecendo ao longo do tempo, ressaltando ora um aspecto, uma metodologia, determinado referencial teórico, o monitoramento dessa produção deve ser paralelamente elaborado, de acordo com Soares e Maciel (2000). Pois, ao identificar e explicitar os caminhos que a ciência percorreu, tornase possível revelar o processo de construção do conhecimento sobre determinado tema, ou seja, identificam-se duplicações, contradições e, especialmente, lacunas, isto é, aspectos ainda não explorados.

Ao que parece, "em tudo o que se refere à ciência, os indicadores bibliométricos e cienciométricos tornaram-se essenciais" (MACIAS-CHAPULA, 1998, p. 134). Assim, a avaliação do conhecimento possibilita, para Vanti (2002), dignificar o saber quando há utilização de métodos confiáveis e sistemáticos, permitindo revelar à sociedade como tal conhecimento foi-se desenvolvendo e de que maneira "tem contribuído para resolver os problemas que se apresentam dentro de sua área de abrangência" (p. 152). Portanto, "parece clara a importância de se dispor de uma distribuição que nos informe sobre o número de autores, trabalhos, países ou revistas que existem em cada categoria de produtividade, utilidade ou o que mais desejarmos saber" (PRICE, 1976, p. 39). 
Os indicadores da atividade científica parecem ser, portanto, o cerne dos debates atualmente, tanto na perspectiva das relações entre o avanço da ciência e da tecnologia como no progresso econômico e social (MACIAS-CHAPULA, 1998; MUGNAINNI; JANNUZZI; QUONIAM, 2004; SILVA; SANTOS; RODRIGUES, 2011; RAMOS; FARIA; FARIA, 2014). Assim, os estudos de natureza bibliométrica são fontes de grande proveito e fecundidade para se conhecer e analisar um campo científico relatam Araújo e Alvarenga (2011, p. 56). Os autores ainda complementam que:

As publicações se constituem em instâncias privilegiadas para o estudo do comportamento de dada disciplina ou campo científico, sob os mais variados aspectos, respondendo diferentes questões: quais são as frentes de pesquisas desse campo, considerando-se diferentes variáveis, pesquisadores/autores, instituições ou temas; quais são os padrões de comunicação entre seus pares, tais como os tipos de canais preferidos e as parcerias; quais são as bases epistemológicas em que se fundamentam suas pesquisas: autores, títulos clássicos, línguas, países, datas, dentre outras.

A informação sempre foi um insumo importante para o gerenciamento dos negócios. Nesse sentido, em função do crescimento do agronegócio, da competitividade e, por consequência, da complexidade da agricultura brasileira nos últimos anos, o conhecimento se tornou uma ferramenta ainda mais essencial (MAPA, 2007). Deste modo, avaliar as produções científicas relativas ao agronegócio traduz-se em apoio para o desenvolvimento deste setor.

\section{METODOLOGIA}

Dada a importância dos indicadores da atividade científica, os meta-estudos surgiram e se multiplicaram, proporcionando, não somente a consolidação da Engenharia de Produção enquanto área de conhecimento, mas o surgimento de indagações pertinentes à sua própria produção, conforme salientam Romanowski e Ens (2006).

Os critérios de seleção dos artigos para este estudo seguiram os procedimentos de coleta e de análise definidos por Tonelli et al. (2003), que 
consideraram os mais citados meta-estudos, a exemplo de Vieira (1998) e Perin et al. (2000).

As bases de dados escolhidas como fontes de pesquisa para esta discussão foram: anais do Encontro Nacional de Engenharia de Produção/ENEGEP (o ENEGEP é o principal evento científico da área, realizado desde 1981, conta com o apoio da Associação Brasileira de Engenharia de Produção/ABEPRO, e tem registrado crescimento de participação de profissionais e estudantes a cada edição); e revistas classificadas no Qualis/Capes 2014 de Engenharia III no estrato B5 ou superior, contanto que fossem periódicos genuínos da área de Engenharia de Produção, o que exclui revistas de outras áreas ou multidisciplinares. O quadro 1 apresenta os periódicos pesquisados e sua respectiva classificação no Qualis/Capes 2014.

Quadro 1. Lista de periódicos pesquisados

\begin{tabular}{|c|l|c|}
\hline $\mathbf{N}^{\circ}$ & Periódico & Qualis/CAPES 2014 \\
\hline $\mathbf{1}$ & Produção (São Paulo. Impresso) & B2 \\
\hline $\mathbf{2}$ & Gestão \& Produção & B3 \\
\hline $\mathbf{3}$ & Revista Produção Online & B4 \\
\hline $\mathbf{4}$ & Produto \& Produção (Online) & B4 \\
\hline $\mathbf{5}$ & Product (IGDP) & B4 \\
\hline $\mathbf{6}$ & Exacta (Online) & B4 \\
\hline $\mathbf{7}$ & GEPROS. Gestão da Produção, Operações e Sistemas & B4 \\
\hline $\mathbf{8}$ & Pesquisa Operacional para o Desenvolvimento & B4 \\
\hline $\mathbf{9}$ & Brazilian Journal of Operations and Production Management & B4 \\
\hline $\mathbf{1 0}$ & Iberoamerican Journal of Industrial Engineering & B4 \\
\hline $\mathbf{1 1}$ & Revista Gestão Industrial & B5 \\
\hline $\mathbf{1 2}$ & INGEPRO - Inovação, Gestão e Produção & B5 \\
\hline $\mathbf{1 3}$ & Pesquisa \& Desenvolvimento em Engenharia de Produção & B5 \\
\hline $\mathbf{1 4}$ & S \& G. Sistemas \& Gestão & B5 \\
\hline $\mathbf{1 5}$ & Independent Journal of Management \& Production & B5 \\
\hline
\end{tabular}

Fonte: Elaboração dos autores. 
A definição do corpus seguiu a regra da exaustividade de Bardin (2011). Nesse sentido, não foi estabelecido um corte temporal, a priori, para validar os artigos recuperados na busca. Todos os textos que emergiram a partir dos critérios de busca estabelecidos foram considerados válidos. O critério de busca levou em consideração categorias analíticas que deveriam constar como palavras-chave no artigo: Manga, Mangicultura, Vale do São Francisco, Vale das Frutas, Agricultura, Agronegócio. Nesse sentido, totalizaram seis artigos completos e selecionados.

Cada artigo foi analisado em termos de seu conteúdo com a finalidade de identificar informações a respeito dos aspetos acima indicados. Os artigos estão identificados, quando necessário, ao longo das análises de acordo com a simbologia adotada no Quadro 2 abaixo.

Quadro 2. Simbologia para identificação dos artigos

\begin{tabular}{|l|c|}
\hline & Identificação dos artigos \\
\hline Revista Gestão \& Produção & G\&P_2009 \\
\hline Revista Produção Online & PROD.ONLINE_2015 \\
\hline \multirow{2}{*}{ ENEGEP - Encontro Nacional de Engenharia de Produção } & ENEGEP_2009 \\
\cline { 2 - 2 } & ENEGEP_2011 \\
\cline { 2 - 2 } & ENEGEP_2012 \\
\cline { 2 - 2 } & ENEGEP_2014 \\
\hline
\end{tabular}

Fonte: Elaboração dos autores.

Como técnica de análise, optou-se pela análise de conteúdo (AC) apresentada por Bardin (2011, p. 44):

conjunto de técnicas de análise das comunicações que utiliza procedimentos sistemáticos e objetivos de descrição do conteúdo das mensagens. A intenção da análise de conteúdo é a inferência de conhecimentos relativos às condições de produção, inferência esta que recorre a indicadores (quantitativos ou não).

A seção a seguir apresenta a análise dos artigos recuperados. 


\section{RESULTADOS E DISCUSSÕES}

Somente foram encontrados artigos com foco na temática abordada em dois periódicos, quais sejam: Revista Gestão \& Produção (G\&P_2009) e Revista Produção Online (PROD.ONLINE_2015); e em quatro edições do ENEGEP (ENEGEP_2014, ENEGEP_2012, ENEGEP_2011, ENEGEP_2009).

Ressalta-se que apenas um, dos seis artigos recuperados para análise, apresenta temática exclusiva para a mangicultura no Vale do São Francisco. Os demais artigos apresentam uma abordagem para manga e uva no Vale do São Francisco (03 artigos) e manga e outras frutas no Vale do São Francisco e no Brasil (02 artigos). Importa ressaltar também que os textos não abordam a região como Vale das Frutas, mas ainda trazem a denominação de Vale do São Francisco ou Submédio do São Francisco. Esta classificação utilizada pelos autores dos textos analisados permite dizer que ainda existe uma certa miopia na análise da realidade da produção do agronegócio da região, conforme destaca Cassundé Junior (2015). É importante destacar que apenas mencionar Polo Petrolina/Juazeiro ou Vale do São Francisco é insuficiente para responder pela abrangência do agronegócio da região. No entanto, também não é legitimo dizer que a região que envolve o agronegócio é o Submédio do Rio São Francisco, pois o Submédio do Rio São Francisco envolve outras áreas que não apenas a do agronegócio destinada à produção de frutas (a exemplo o Oeste baiano, com produção de soja). Isto posto, para efeitos desse estudo, defendese que a região produtora de frutas, sustentada pela irrigação do Rio São Francisco, deva ser denominada como Vale das Frutas. Assim, como sugere Cassundé Junior (2015), os trabalhos mais recentes devem adotar a terminologia Vale das Frutas em substituição a Vale do São Francisco, Polo Petrolina/Juazeiro ou Submédio do São Francisco.

Outros aspectos chamam bastante atenção: as publicações sobre o tema são recentes (começaram a partir de 2009), não são constantes (não foram encontrados estudos publicados em 2010, 2013 e 2016, por exemplo) nem, tampouco, expressivas em termos de quantidade por evento ou edição do periódico (todas as edições/ eventos têm apenas um artigo publicado). Isso implica dizer que, embora o Vale tenha recebido mais atenção a partir do grau de inserção internacional desse setor 
desde a política nacional de abertura comercial em 1990 (BUSTAMANTE, 2009), poucos foram os estudos encontrados em Engenharia de Produção que procuram compreender a produção da mangicultura do ponto de vista da Engenharia de Produção.

Entre autores e coautores, foi possível identificar um total de 11 pesquisadores. Chama atenção o fato de que apenas um dos coautores era aluno na época da publicação do texto, todos os demais são docentes de carreira. A análise de autoria dos artigos permite mostrar que todos os trabalhos foram elaborados em sistema de parceira, sendo especialmente significativa a quantidade de artigos que apresentam um autor e um coautor. Dadas as pressões do sistema de avaliação da Capes, que atribui importância decisiva à pesquisa que resulte em publicações por publicação, os pesquisadores que optam pela coautoria aumentam, portanto, suas possibilidades de publicação, conforme destacam Cassundé, Barbosa e Mendonça (2016).

Com relação à instituição de origem dos pesquisadores, merece destaque o fato de que a produção científica se concentra em pesquisadores lotados em instituições do Nordeste, quais sejam: Universidade Federal do Vale do São Francisco (UNIVASF), Universidade Federal de Pernambuco (UFPE), Universidade Federal Rural de Pernambuco (UFRPE) e Universidade Federal da Bahia (UFBA). Notadamente a UNIVASF apresenta o maior número de pesquisadores (cinco pesquisadores, vinculados ao Colegiado de Engenharia de Produção) com publicação. Atribui-se este maior interesse sobre o tema em razão da localidade em que tal universidade está inserida e ao propósito a que se destina, uma vez que a UNIVASF, com sede na cidade de Petrolina (Pernambuco), tem o semiárido nordestino e o Vale do São Francisco como referenciais de atuação, uma região abrangida por um raio de 250 quilômetros, "centrado nas cidades de Petrolina e Juazeiro, englobando um total de 74 municípios em três estados do Nordeste, sendo 35 na Bahia, 30 em Pernambuco e 9 no Piauí" (UNIVASF, 2008, p. 13).

Com relação às referências utilizadas, foram catalogadas 141 obras nos seis textos analisados. O procedimento adotado foi o de registrar a frequência em que cada autor aparecia como referência em cada artigo. A análise das referências permite afirmar que grande parte da literatura que apoiou esses estudos é de 
origem europeia. Duas instituições também foram bastante utilizadas como fonte de referência: o IBRAF - Instituto Brasileiro de Frutas (Nacional) e a FAO - Food and Agriculture Organization of the United Nations (Internacional). É importante dizer que ambas as instituições se fundamentam em princípios que visam contribuir, de modo direto ou indireto, para o desenvolvimento do agronegócio. Todos os principais autores referenciados são de origem estrangeira, subdividindo-se entres as áreas de estatística, economia, sociologia e engenharia de produção. Com relação às obras mais referenciadas, 37,04\% possuem origem nacional e 62,96\% estrangeira. Os principais autores/instituições estão indicados, por ordem decrescente de utilização, no Quadro 3 abaixo.

Quadro 3. Principais autores referenciados

\begin{tabular}{|l|c|c|}
\hline \multicolumn{1}{|c|}{ Autor/Instituição } & Origem & $\begin{array}{c}\text { Quantidade de } \\
\text { citações }\end{array}$ \\
\hline Robert F. Engle & EUA & 4 \\
\hline Matteo Kalchschmidt & Itália & 4 \\
\hline George Edward Pelham Box & Inglaterra & 4 \\
\hline Pamela Danese & Itália & 3 \\
\hline $\begin{array}{l}\text { FAO - Food and Agricultural Organization of the United } \\
\text { Nations }\end{array}$ & EUA & 3 \\
\hline IBRAF - Instituto Brasileiro de Frutas & Brasil & 3 \\
\hline John Humphrey & Inglaterra & 3 \\
\hline
\end{tabular}

Fonte: Elaboração dos autores.

Os periódicos Gestão \& Produção e Produção Online e o evento do ENEGEP possuem, separadamente, 10 áreas de atuação da Engenharia de Produção, onde estão distribuídas as publicações encontradas. Os seis artigos em análise possuem o foco ou na área da Gestão/Gerência da Produção ou na Pesquisa Operacional. O Quadro 4 apresenta as informações relativas à área e escopo de cada artigo analisado. Os artigos estão dispostos em ordem crescente de ano de publicação. 
Quadro 4. Artigo x área de publicação x escopo da pesquisa

\begin{tabular}{|l|l|l|}
\hline \multicolumn{1}{|c|}{ Artigo } & \multicolumn{1}{c|}{ Área } & \multicolumn{1}{c|}{ Escopo da pesquisa } \\
\hline ENEGEP_2009 & Pesquisa Operacional & Decisão multicritério \\
\hline G\&P_2009 & Gestão da Produção & Cadeia produtiva \\
\hline ENEGEP_2011 & Gestão da Produção & $\begin{array}{l}\text { Logística e gestão da cadeia de suprimentos e } \\
\text { distribuição }\end{array}$ \\
\hline ENEGEP_2012 & Gestão da Produção & $\begin{array}{l}\text { Logística e gestão da cadeia de suprimentos e } \\
\text { distribuição }\end{array}$ \\
\hline ENEGEP_2014 & Pesquisa Operacional & Análise de demandas por produtos \\
\hline PROD.ONLINE_2015 & Pesquisa Operacional & Análise de demandas por produtos \\
\hline
\end{tabular}

Fonte: Elaboração dos autores.

Considerando a importância das palavras-chave para recuperação dos artigos científicos, foi estabelecida uma nuvem de palavras (Figura 1) a partir dos termos utilizados enquanto instrumento de representação da informação contida nos textos analisados. É importante salientar, neste ponto, que as nuvens de palavras são, conforme Rivadeneira et al. (2007), apresentações visuais de um conjunto de palavras selecionadas por alguma lógica, em que os atributos do texto, tais como tamanho ou a cor são usados para representar certas características, como, por exemplo, a frequência. Foram, ao todo, 22 termos utilizados como representação dos estudos, dos quais 17 palavras-chave sem repetição. As palavras-chave estão representadas de acordo com sua frequência na nuvem de palavras da Figura 1. 


\section{manga-orgânica \\ modelos-ARIMA \\ MOdelOS-GARCF mevrnanga \\ séries-temporais frutas-in-natura \\ canais-distribuição comercializaçāo-frutas \\ VI. análise-multicritéria

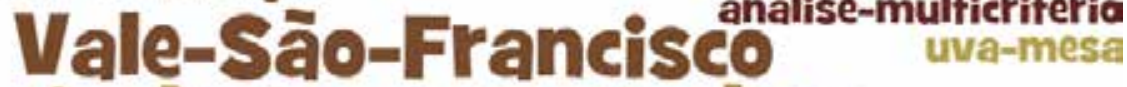 \\ fruticultula-exportadora \\ cadeja-suprimentos previsão \\ mercados-futuros \\ supermercados}

Figura 1. Nuvem de palavras para as palavras-chave utilizadas

Fonte: Elaboração dos autores.

A partir da nuvem de palavras, e da análise do escopo de cada um dos textos, foi possível identificar os objetivos e abrangências das pesquisas. Importa dizer que pouco é estudado sobre a cadeia produtiva da manga no Vale das Frutas. Os estudos estão concentrados na elaboração de previsão de modelos econométricos para a exportação e análise de séries temporais da produção frutífera. Nesse sentido, sugere-se uma agenda de pesquisas futuras em que sejam contemplados também outros aspectos da mangicultura, tais como: logística e logística reversa, qualidade, ergonomia e segurança do trabalho na produção de mangas, engenharia de produto, gestão ambiental e responsabilidade social dos produtores locais.

O Quadro 5 apresenta o perfil metodológico e lócus dos artigos (os artigos estão dispostos em ordem crescente de ano de publicação). Os artigos publicados são predominantemente de natureza teórico-empírica e já se verifica uma utilização de metodologias qualitativas para análise. Nesse sentido, pode-se dizer que apesar das publicações serem da área de engenharia, o que se espera uma maior ênfase no uso de ferramentas e métodos matemáticos/quantitativos, pode demonstrar 
uma mudança no perfil dos pesquisadores, que passa a considerar a vertente da Engenharia de Produção que concentra diversas técnicas qualitativas de gestão, análise e resolução de problemas.

Quadro 5. Perfil metodológico

\begin{tabular}{|l|c|c|}
\hline Artigo & Metodologia & Natureza \\
\hline ENEGEP_2009 & Quantitativa & Teórico-empírica \\
\hline G\&P_2009 & Qualitativa & Teórico-empírica \\
\hline ENEGEP_2011 & Qualitativa & Teórico-empírica \\
\hline ENEGEP_2012 & Qualitativa & Teórico-empírica \\
\hline ENEGEP_2014 & Quantitativa & Teórico \\
\hline PROD.ONLINE_2015 & Quantitativa & Teórico \\
\hline
\end{tabular}

Fonte: Elaboração dos autores.

É interessante considerar que diversos conceitos, filosofias e metodologias enfatizados no curso de Engenharia de Produção são aplicados e desenvolvidos dentro do âmbito do agronegócio. Os campos e possibilidades de atuação dentro do agronegócio, fruticultura e mangicultura são inúmeros, podendo estes serem explorados em artigos posteriores, no intuito de ampliar as perspectivas de abordagem dos autores de artigos científicos. Assim como expandir os olhares das empresas que utilizam os meios acadêmicos como fonte de conhecimento e inovação organizacional, sobre as habilidades da Engenharia de Produção.

\section{CONCLUSÃO}

Este trabalho se propôs a analisar como a temática da mangicultura no Vale das Frutas vem sendo abordada nos principais periódicos e eventos da área de Engenharia de Produção, no sentido de contribuir para o entendimento de como e quais aspectos estão sendo estudados, pelos acadêmicos, sobre a produção da manga. Por meio da análise dos artigos foi possível identificar, por exemplo, termos mais utilizados nas palavras-chave, os autores que mais publicam e suas instituições de origem, as principais referências utilizadas e os autores mais referenciados. A 
principal contribuição de um estudo como este é o de monitorar periodicamente o alcance das pesquisas desenvolvidas em um período de tempo específico, no sentido de verificar critérios de avanço (ou não) em determinado campo de investigação.

Este trabalho revela que a produção científica brasileira sobre mangicultura no Vale das Frutas na área de Engenharia de Produção é bastante pontual e, em alguns casos, como em periódicos, surpreendentemente inexistente, uma vez que dos 15 periódicos analisados, apenas em dois foram encontrados artigos que satisfizeram os critérios de busca para este estudo. Esse aspecto permite visualizar diversas inquietações entre as quais a mais expressiva delas é: por qual motivo a mangicultura no Vale das Frutas não tem recebido - pelo menos no que tange aos periódicos e aos congressos nacionais de Engenharia de Produção - a atenção merecida?

É importante ressaltar que, em função das limitações deste estudo (considerar apenas as produções científicas em Engenharia de Produção) os resultados aqui apresentados não podem ser generalizados para outros eventos, periódicos ou ainda outras áreas. Os resultados dizem respeito aos padrões dos periódicos e evento analisados, entretanto, por serem academicamente reconhecidos, este trabalho tem condições de contribuir para a compreensão da produção científica sobre mangicultura no Vale das Frutas em Engenharia de Produção, inclusive propondo uma agenda de pesquisas.

É igualmente importante ressaltar que, conforme visto na literatura (FERREIRA, 2002; VANTI, 2002; CARDOSO et al., 2005; ARAÚJO, 2006; ARAÚJO; ALVARENGA, 2011; CASSUNDÉ; CASSUNDÉ JUNIOR, 2012), embora sejam extremamente úteis para a compreensão do desenvolvimento da ciência, especialmente para uma área ainda jovem, como a Engenharia de Produção, os estudos sobre indicadores apresentam uma certa validade. Pois, ao se considerar a dinamicidade e evolução própria da ciência, tais estudos demandam um constante revisar, de forma a continuarem apontando possíveis caminhos de investigação aos pesquisadores da área.

Por fim, conclui-se que a temática Mangicultura no Vale do São Francisco é pouco abordada em publicações acadêmicas, tanto em periódicos como no ENEGEP, o que identifica a necessidade de geração de maiores conhecimentos nesta área, visto a sua importância para a região. 


\section{REFERÊNCIAS}

ALVES, J. M. Competitividade e tendência da produção de manga para exportação do nordeste do Brasil. 2002. Tese (Doutorado) - Escola Superior de Agricultura Luiz de Queiroz, ESALQ, Programa de Pós-Gradução em Economia Aplicada, Piracicaba, SP.

ARA - Ara Empreendimentos. Ara Agrícola. 2015. Disponível em: < http://www. araempreendimentos.com.br/\#/negocios >. Acesso em: 12 jan. 2015.

ARAÚJO, C. A. Bibliometria: evolução histórica e questões atuais. Em Questão, Porto Alegre, v. 12, n. 1, p. 11-32, jan./jun. 2006.

ARAÚJO, R. F.; ALVARENGA, L. A bibliometria na pesquisa científica da pós-graduação brasileira de 1987 a 2007. Ciência da Informação, Florianópolis, v. 16, n. 31, p. 51-70, 2011.

BANCO DO NORDESTE. Rede de Irrigação. Doe. 13. Fortaleza, 1999.

BARBOSA, P. M. S.; FERNANDES, L. R. R. M. V.; LAGE, C. L. S. Quais são as Indicações Geográficas brasileiras? Revista Brasileira de Pós-Graduação, v. 10, p. 317-347, 2013.

BARDIN, L. Análise de conteúdo. São Paulo: Edições 70, 2011.

BUSTAMANTE, P. M. A. C. A fruticultura no Brasil e no Vale do São Francisco: vantagens e desafios. Revista Econômica do Nordeste, v. 40, p. 153-171, 2009.

CARDOSO, R. L.; MENDONÇA NETO, O. R.; RICCIO, E. L.; SAKATA, M. C. G. Pesquisa científica em contabilidade entre 1990 e 2003. Revista de Administração de Empresas, São Paulo, v. 45, n. 2, p. 34-45, abr/jun 2005.

CASSUNDÉ, F. R.; BARBOSA, M. A. C.; MENDONÇA, J. R. C. A influência da tradição anglo-saxônica nos estudos organizacionais brasileiros: o que mudou (ou não) nos últimos 15 anos? Perspectivas em Gestão \& Conhecimento, João Pessoa, v. 6, n. 1, p. 238-254, jan./jun. 2016. 
CASSUNDÉ, F. R.; CASSUNDÉ JUNIOR, N. O estado do conhecimento sobre educação a distância (EAD) em Administração: por onde caminham os artigos? Revista Gestão e Planejamento, Salvador, v. 13, n. 2, p. 366-374, maio/ago. 2012.

\section{CASSUNDÉ JUNIOR, N. F. Internacionalização e Dimensões organizacionais:} Proposta de um Framework Teórico-Dinâmico. Tese (Doutorado) - Universidade Federal de Pernambuco, UFPE, Programa de Pós-Gradução em Administração, Recife, 2015.

CASSUNDÉ JUNIOR, N. F.; LIMA, R. C.; PIMENTEL, C. R. M. As potencialidades e distorções comerciais no mercado internacional da mangicultura brasileira. In: SOBER - CONGRESSO BRASILEIRO DE ECONOMIA E SOCIOLOGIA RURAL, 44., 2006, Fortaleza. Questões agrárias, educação no campo e desenvolvimento, Anais... 2006.

CBHSF - Comitê da Bacia Hidrográfica do Rio São Francisco. Municípios do Submédio SF. 2012. Disponível em: http://cbhsaofrancisco.org.br/municipios-dosubmedio-sf/. Acesso em: 28 ago. 2014.

COANA - Cooperativa Agrícola Nova Aliança. A Coana. 2015. Disponível em: < http:// www.coanabr.com.br/principal/> . Acesso em: 12 jan. 2015.

CODEVASF. Elenco de Projetos. 2015. Disponível em: < http://www.codevasf.gov. br/principal/perimetros-irrigados/elenco-de-projetos > . Acesso em: 15 maio. 2015.

CODEVASF. Perímetros irrigados. 2010. Disponível em: <http://www. codevasf.gov.br/principal/perimetros-irrigados/polos-de-desenvolvimento - 1/juazeiro-petrolina >. Acesso em: 15 maio. 2015.

EMBRAPA. Cultivo da Mangueira: socioeconomia. 2010. Disponível em: $\quad$ https://sistemasdeproducao.cnptia.embrapa.br/FontesHTML/Manga/ CultivodaMangueira_2ed/socioeconomia.htm. Acesso em: 13 jun. 2016.

EMBRAPA. Subsídios técnicos para a indicação geográfica de procedência do Vale do Submédio São Francisco: uva de mesa e Manga. Petrolina: Embrapa SemiÁrido, 2009. 
EXSA - EXSA (PTY) LTD. Arnaldo Eijsink from GRUPO JD in Brazil visits IFG evaluation block. 2013. Disponível em: < http://www.zoominfo.com/CachedPage/? archive_id=0\&page_id=6596866032\&page_url=//www.exsa.com/_blog/Latest News/post/Arnaldo_Eijsink_from_GRUPO_JD_in_Brazil_visits_IFG_evaluation_ block/\&page_last_updated $=$ 2013-05-24T06:58:23\&firstName $=$ Arnaldo\&lastName $=$ Eijsink $>$. Acesso em: 28 ago. 2013.

FERREIRA, N. S. A. As pesquisas denominadas "estado da arte". Educação \& Sociedade, ano XXIII, n. 79, ago./2002.

FREITAS, E. M.; RUPOLO, M.; OLIVEIRA, B. R. B. Processo de internacionalizacao de uma empresa do vale do São Francisco: influência dos agentes externos e das escolhas gerenciais. InternexT, v. 9, n. 1, p. 40, 2014.

GALVÃO, A. S. S. Ciclos econômicos recentes e perspectivas para a região do submédio Vale do São Francisco com ênfase na fruticultura irrigada. 2010. Dissertação (Mestrado Profissional em Economia Aplicada) - Universidade Federal de Pernambuco, Programa de Pós-Graduação em Economia, Recife, PE.

IBGE. Produção Agrícola Municipal. Prod. agric. munic., v. 39, Rio de Janeiro, 2012.

JESUS JUNIOR, C.; SIDONIO, L.; MORAES, V. E. G. Fruticultura: formas de organização nos principais países exportadores. BNDES Setorial, 4, p. 239-270. 2011.

LANDIM, M. F. M.; ALENCAR, M. T. Urbanização e Agronegócio: Petrolina, a cidade em cena. Revista Equador, v. 1, p. 4-22, 2013.

LOPES, P. R. C.; OLIVEIRA, I. V. M. Produção de pera no Vale do São Francisco. In: REUNIÃO TÉCNICA DA CULTURA DA PEREIRA, 2012, LAGES, PRODUÇÃO DE PERA NO VALE DO SÃO FRANCISCO. Anais... LAGES: UDESC, 2012. p. 56-65.

MACIAS-CHAPULA, C. A. O papel da informetria e da cienciometria e sua perspectiva nacional e internacional. Ciência da Informação, Brasília, v. 27, n. 2, p. 134-140, maio/ago. 1998. 
MAPA, 2007. Ministério da Agricultura, Pecuária e Abastecimento. (Série Agronegócios: Cadeia Produtiva de Frutas). Disponível em: <http://www. ibraf.org.br/x_files/Documentos/Cadeia_Produtiva_de_Frutas_S\%C3\%A9rie_ Agroneg\%C3\%B3cios_MAPA.pdf) > . Acesso em: 22 jun. 2016.

MAPA, 2012. Informativo sobre a Estiagem no Nordeste - $\mathrm{n}^{\mathrm{o}} 12$ de 03/08/2012. Disponível em: <http://www. agricultura.gov.br/arq_editor/file/Homepage/Combate\% 20a\%20Seca/Informativo\%20estiagem\%20NE\%20n\%C2\%BA\%2012.pdf>. Acesso em: 11 out. 2014.

MARIANO, J. L. A eficiência dos colonos na agricultura irrigada no Vale do São Francisco: uma análise comparativa de modelos de fronteira paramétrica e não paramétrica. 1999. Tese (Doutorado) - Universidade Federal de Pernambuco, Programa de Pós-Graduação em Economia-PIMES, Recife, PE.

MATTOSINHO, C. M. S.; HORA, G. B.; SANTOS, M. R. F.; XAVIER, M. G. P. Desempenho do Beneficiamento da Uva de Mesa: Um Estudo de Caso na Região do Submédio São Francisco. In: CONGRESSO DA SOCIEDADE BRASILEIRA DE ECONOMIA, ADMINISTRAÇÃO E SOCIOLOGIA RURAL - SOBER, 51., 2013, Belém. Anais... Belém: SOBER, 2013.

MUGNAINI, R.; JANNUZZI, P. M.; QUONIAM, L. Indicadores bibliométricos da produção brasileira: uma análise a partir da base Pascal. Ciência da Informação, Brasília, v. 33, n. 2, p. 123-131, maio/ago. 2004.

OECD-FAO. OECD-FAO Agricultural Outlook. OECD Publishing, Paris. 2015. Disponível em: < http://www.fao.org/3/a-i4738e.pdf> . Acesso em: 12 mar. 2015.

OLIVEIRA, E. M.; OLIVEIRA, R. S. Novas territórialidades no espaço rural irrigado de Petrolina-PE: uma análise do uso da força de trabalho na produção e desenvolvimento do agronegócio. In: ENCONTRO NACIONAL DE GEÓGRAFOS, 17., 2012, Entre escalas, poderes, ações, Geografias, Belo Horizonte (MG). Anais... , Belo Horizonte [s.n.], 2012. 
PERIN, M. G. et al. A pesquisa survey em artigos de marketing nos ENANPADs da década de 90. In: ENCONTRO DOS PROGRAMAS DE PÓS-GRADUAÇÃO EM ADMINISTRAÇÃO (ENANPAD), 24., 2000, Florianópolis. Anais... Florianópolis: ANPAD, 2000.

PIMENTEL, C. R M.; ALVES, R. E.; FILGUEIRAS, H. A. C. Mercado internacional de manga: situação atual e perspectivas. In: PIMENTEL, C. R. M. et al. Frutas do Brasil: Manga pós-colheita. Fortaleza: Embrapa Agroindústria Tropical, 2000.

PRICE, D. S. O desenvolvimento da ciência: análise histórica, filosófica, sociológica e econômica. Rio de Janeiro: Livros Técnicos e Científicos, 1976.

QUEIROZ, M. A.; BARROS, L. M.; CARVALHO, L. P.; CANDEIA, J. A.; FERRAZ, E. Plant breeding in the semiarid region of Brazil: examples of success. Crop Breeding and Applied Biotechnology, 2012, v. 12, n. spe, p. 57-66.

RAMOS, A.; FARIA, P. M.; FARIA, Á. Revisão sistemática de literatura: contributo para a inovação na investigação em Ciências da Educação. Revista Diálogo Educacional, Curitiba, v. 14, n. 41, p. 17-36, jan./abr. 2014.

RIBEIRO, K. Á.; NASCIMENTO, D. C.; CASSUNDÉ JUNIOR, N. F.; MORATO, J. A. Q. APL como uma estratégia de potencializar as fronteiras mercadológicas do apicultor no perímetro de irrigação Sen. Nilo Coelho em Petrolina/PE. Revista de Gestão, Finanças e Contabilidade, v. 3, p. 99-120, 2013.

ROMANOWSKI, J. P.; ENS, R. T. As pesquisas denominadas do tipo "estado da arte" em educação. Revista Diálogo Educacional, v. 6, n. 19, p. 37-50, set./dez. 2006.

SANTOS, C. E. et al. Anuário Brasileiro da Fruticultura - 2014 - ABF, 2014. Santa Cruz do Sul: Gazeta Santa Cruz, 2014. Disponível em: <http://www. grupogaz.com.br/tratadas/eo_edicao/4/2014/03/20140325_3d8463877/pdf/4333_ fruticultura_2014.pdf >. Acesso em: 04 jul. 2016.

SILVA, P. C. G. Articulação dos interesses públicos e privados no pólo PetrolinaPE/Juazeiro-BA: em busca de espaço no mercado globalizado de frutas frescas. Tese (Doutorado) - Universidade Estadual de Campinas, 2001. 
SILVA, R. A.; SANTOS, R. N. M.; RODRIGUES, R. S. Estudo bibliométrico na base LISA: um enfoque nos artigos sobre surdos. Em Questão, Porto Alegre, v. 17, n. 1, p. 289-304, jan./jun. 2011.

SILVA-MATOS, R. R. S.; LOPES, P. R. C.; SOUZA, G. M. M.; OLIVEIRA, I. V. M. Racionalização do Uso de Agrotóxicos na Produção Integrada de Manga no Submédio do Vale do São Francisco. Bioscience Journal, v. 30, p. 372-379, 2014.

SILVA, T. G. F.; ZOLNIER, S.; MOURA, M. S. B.; CARMO, J. F. A.; RIBEIRO, A. Fator de desacoplamento em um canavial irrigado no submédio do Vale do São Francisco. Revista Brasileira de Engenharia Agrícola e Ambiental, v. 16, n. 8, 2012.

SIQUEIRA, T. V. A cultura da manga: desempenho no período 1961/2001. Rio de Janeiro: BNDES, 2003.

SNA. Sociedade Nacional da Agricultura. Indicação Geográfica Vale do Submédio São Francisco. 2015. Disponível em: < http://sna.agr.br/indicacao-geografica-valedo-submedio-sao-francisco/> . Acesso em: 04 jul. 2016.

SOARES, M. B.; MACIEL, F. Alfabetização. Brasília: MEC/INEP/COMPED, 2000.

TONELLI, M. J. et al. Produção acadêmica em recursos humanos no Brasil: 19912000. Revista de Administração de Empresas, São Paulo, v. 43, n. 1, jan./mar. 2003.

TREICHEL, M. et al. Anuário Brasileiro da Fruticultura 2016 - ABF, 2016. Santa Cruz do Sul: Gazeta Santa Cruz, 2016. Disponível em: <http://www.grupogaz.com. br/tratadas/eo_edicao/4/2016/04/20160414_0d40a2e2a/pdf/5149_2016fruticultura. pdf > . Acesso em: 14 jun. 2016.

UNIVERSIDADE FEDERAL DO VALE DO SÃO FRANCISCO. Projeto pedagógico do curso de Administração. Petrolina, 2008.

VANTI, N. A. P. Da bibliometria à webometria: uma exploração conceitual dos mecanismos utilizados para medir o registro da informação e a difusão do 
conhecimento. Ciência da Informação, Brasília, v. 31, n. 2, p. 152-162, maio/ago. 2002.

VIEIRA, F. G. D. Por quem os sinos dobram? Uma análise da publicação científica na área de marketing do Enanpad. In: ENCONTRO DOS PROGRAMAS DE PÓSGRADUAÇÃO EM ADMINISTRAÇÃO (ENANPAD), 22, 1998, Foz do Iguaçu. Anais... Foz do Iguaçu: ANPAD, 1998.

VITTI, A.; SEBASTIANI, R. E. G.; VICENTINI, C. A.; BOTEON, M. Perspectivas da fruticultura brasileira exportadora frente aos novos investimentos. Palestra In: SOBER, 2004.

Recebido em: 27 setembro de 2016 Aceito em: 01 de março de 2017 